\title{
41
}

\section{Information technology capability-how does it develop?}

\author{
Steve Kennewell \\ University College of Swansea \\ United Kingdom
}

\begin{abstract}
This paper examines the nature of skills in applying Information Technology in terms of the relation between the conceptual and procedural knowledge involved. A number of scenarios drawn from the author's recent classroom studies is presented in order to illustrate common difficulties which school students have in progressing beyond very basic skills in the use of generic applications.

A simplified theory of conceptual change during active learning, based on cognitive science explanations of conceptual development and on humancomputer interaction research, is set out in order to help explain these phenomena. Conclusions are drawn concerning the use of analogy and cognitive conflict in teaching IT.
\end{abstract}

Main conference themes: informatics as study topic

Educational areas: primary education, secondary education

Study topics: computer literacy

Secondary keywords: basic skills, classroom practice, cognition, learning models, teaching methods 


\section{INTRODUCTION}

\section{What is IT capability?}

The orders for Technology in the National Curriculum for England and Wales [1] require that pupils should be able to use information technology to:

- communicate and handle information;

- design, develop and evaluate models of real or imaginary situations;

- measure and control physical variables and movement.

They should be able to make informed judgements about the application and importance of information technology, and its effect on the quality of life. These abilities are termed IT Capability. According to Birnbaum [2], IT capability is developed by

"pursuing worthwhile tasks in a variety of contexts, the contexts and tasks being chosen to facilitate the development of skills, knowledge, concepts and values necessary in the various areas of competency required."

Birnbaum further suggests that the curriculum as a whole provides suitable contexts for such tasks, and that IT can be learned while pupils are working on other subjects. IT Capability thus comprises knowledge of what IT can achieve, the concepts needed to relate this knowledge to particular tasks, the skills needed to carry out tasks and the values needed to judge whether the application of IT is appropriate. It covers the use of software tools such as word processing, database management, graphics and spreadsheet modelling.

While there is a considerable body of research concerning the teaching and learning of computer programming, much less is known about the development of children's skills with the higher level tools. I shall draw on formal and informal observations from two recent projects to illustrate some problems in developing children's IT skills beyond a very basic threshold within a permeated IT curriculum.

\section{Examples of observed phenomena}

A number of features of children's work with computers in the classroom have been observed repeatedly during recent research on differences in IT learning between primary and secondary schools [3] and in current pilot work on the development of algebraic concepts through computer modelling activities. These features are illustrated by the following phenomena:

- When using a word processor children who used block operations for other editing tasks, deleted large amounts of text by repeated pressing of the delete key rather than using a block delete operation. 
- Again, when using a word processor children who wanted to produce a narrow column of text, pressed the Return/Enter key when they had placed sufficient text on each line, rather than changing the margin settings.

- When using a graphics program to produce a magazine illustration children to whom the straight line tool was familiar, attempted to produce straight lines freehand.

- When using a spreadsheet program children calculated values manually and entered them as data rather than entering a formula.

The frequency and persistence of these features are such that some explanation is required in terms of cognitive processes. Each of them seems to typify a general aspect of difficulty in developing IT capability and explaining these may help to provide some principles on which we can plan future teaching approaches. Cardinale [4] identifies further instances of error, and suggests how the teacher may probe the student's mental model of the system being used. I shall use this well-established concept as the basis of a simplified theoretical framework for the learning and application of IT, then examine the applicability of the theory to the difficulties identified, and finally draw conclusions concerning teaching approaches in primary and secondary education.

\section{Mental models}

Much has been written about mental models for understanding physical processes and human-computer interaction. There is no clear agreement on a simple definition of a mental model, but I will proceed on the basis that it is:

- a person's representation in memory of a system, process or object;

- intended to have the same structure as the real system, though inaccurately;

- incomplete in its representation;

- used to predict the behaviour of the system under known conditions;

- used to guide decision making in interactions with the system;

- not directly accessible to an external observer.

Thus a person's mental model of a computer system contains an understanding of what a computer and its software can do, indicates whether it will be useful to them in a particular situation, and guides their actions when using a computer for a task.

We now need to consider how teachers might use knowledge of mental models when children learn to use IT tools for dealing with tasks. I propose to consider two aspects of the students' representation of their environmentconceptual and procedural (broadly following Anderson [5]). The conceptual representation (CR) is declarative and corresponds to knowing about the 
system. It can be used to predict what the system should be able to do and explain why it behaves as it does in a particular situation. It contains many loosely specified elements and relationships which develop with experience. The procedural representation (PR) comprises a dynamic model of the environment which can be set in action to make predictions about its behaviour according to the state it is in and the action applied to it. This corresponds to knowing how to use the system. The CR represents generic concepts (for example the concept of computer or word processor) as well as specific instances (for example Apple Mac or WordPerfect). The PR is specific to a particular system, but has as an additional feature that it (or parts of it) can be automatized: with sufficient practice certain procedures can be carried out without conscious thought and in parallel with other cognitive activity. The overall model will not be a complete or accurate representation of the system, though clearly it will be more extensive and precise in the case of experts than for novices.

When learning to use a system there are two other models which relate to the learner's mental model [6]. There is a conceptual model of the target system (the model which the teacher or manual is trying to convey) and the teacher's conceptualization of the learner's mental model. Even this does not seem to cover all the interacting representations, however. We might add representations of the task (learner's and teacher's) and of aspects of the domain in which the task is set (that is, the writing process or algebra in our examples). It is also likely that learners with different cognitive styles will develop mental models in different ways. Add to this the potential for differences in mental models amongst a group of learners working together on a task, as is often the case, and we have a highly complex situation to analyze. In this paper, I shall focus only on the learner's mental model.

\section{Modes of learning}

One mode of learning occurs when a PR is not immediately available in the student's memory to indicate how to proceed with an intended action. The student will then use the relationships within the CR to generate a PR which can be enacted. This CR will be based on a combination of experiential, analogical and instructional sources; for a novice a model of an analogous system will be appropriate as basis for development. The teacher may use an analogy explicitly, but in any case the learner is likely draw on concepts which have at least some features in common with the target system. Of course the learner may assume that too many features of the analogous concept transfer and this can cause barriers to learning. We can consider the difference between adult and child learners in this respect. The child whose only analogy is pencil and paper will have a limited starting point for conceptualization of a word 
processor, but will be more receptive to feedback from their experiences - they try out ideas and learn from their mistakes. An adult with a richer store of potential analogies may be able to draw on different ones as appropriate to different aspects of the target system, but may rely too much on inappropriate transfer, for instance from a typewriter.

Experienced interactions with the system will feed back to the conceptual representation which may adjust in accordance with the success or failure of the action. Thus when the procedure is carried out the next time, a new PR may be enacted with improved results. CRs cannot be simply conveyed from an expert to a novice by instruction; they must be constructed by the learner. There seems to be a natural tendency for children to generalize from their experiences (the process named reflective abstraction by Piaget) and then to discriminate the features of their concepts to produce more complex structures in order explain a wider range of phenomena

Another mode of learning is when instruction or demonstration generates a PR directly. This PR may be retained in memory without a change to the structure of the CR, but this is difficult except for very simple procedures. Repeated practice for long term retention will be needed and the PR will be bound to a particular context. This is illustrated by the difficulty which learners, particularly children, find in following step-by-step written instructions. Although secondary teachers use this method to some extent, primary teachers do not see it as a source of learning for their pupils [3].

For effective learning, an adjustment in the CR is necessary. Research in mathematics and science education (for instance Bell [7]) shows that this is most likely to occur in a situation of cognitive conflict when events do not correspond to the prediction made by the learner's mental model, creating a state in which the CR should accommodate to the learner's experiences. But accommodation is rare, however, indicating a high degree of CR stability. Common alternative responses ignore the conflicting evidence, represent the situation as an exceptional case or developing a PR for this situation which remains in conflict with the $\mathrm{CR}$.

The development of the $\mathrm{CR}$ can enable more complex procedures to be grasped and transferred to new contexts where a different PR is needed. Vygotsky's [8] theories of social learning suggest that this will be facilitated by group interaction and other support from peers. However our own observations indicate that learners who rely excessively on their more expert peers, may just be learning a small number of particular procedures by rote rather than developing their CRs. Providing scaffolding by a person with more expertise for a learner can be explained in terms of mental models: a learner will be able to adjust their CR more rapidly if someone with a more extensive and 
accurate CR immediately interprets the learner's errors in relation to their intentions and suggests alternative methods to try.

Learning does not always take place with experience, however. If the target system matches the PR enacted and the results are as expected, no changes will occur to the $\mathrm{CR}$ (though the retention and automatization of the PR may be enhanced). Even if the results of enacting the PR do not produce the expected results, there may be no adjustment of the CR and the learner will treat the result as an aberration often repeating the faulty procedure and blaming the system rather than their representation of it for the error. CRs based on long established beliefs and analogies are particularly difficult to change through instruction or experience.

\section{Effects of mental models on actions}

How do these representations of the system determine the choice of action by the learner when carrying out a task? The learner may have an automatized PR which matches the goal or subgoal subconsciously. This may not be the most effective means of achieving the goal, but its enactment can only be overridden by a metacognitive process or by a constraint imposed by the teacher or the task. If a suitable PR is not available in memory, the learner will use the relationships in the $\mathrm{CR}$ to generate a PR which is expected to bring the learner to his goal. With an interactive system such as a computer the PR does not have to be completely formed before it is enacted since the CR can obtain feedback and cues from the computer to help form or adjust the PR during enactment. When the task goal has to be broken down into subgoals, the cognitive load created by this planning seems to constrain the learner-only the most familiar procedures are likely to be used without cognitive scaffolding, such as prompting by the teacher or carefully structured class discussion.

Children are prime examples of Carroll's [9] active user-when presented with a computer and a task, they will take action of some sort, however unfamiliar and confusing the environment may be. Carroll's production paradox is clearly illustrated in the classroom: the learners' primary goal is the outcome of the task, not the development of new IT skills. If their naive mental models and limited known procedures allow them to reach this goal, then little learning will take place. And as Watson [10] points out teachers often share the goal of outcome production.

\section{Interpretation of observed phenomena and implications for instruction}

The phenomenon concerning word processor block operations can be explained by the automatization of the delete key at an early stage. Which explanation seems more likely than the application of a pencil and paper analogy, for which the use of an eraser or crossing out is rather like a block operation. It is hard to 
create a conflict situation in this case, but in order to stimulate the use of block operations, the teacher could set tasks requiring the deletion of large blocks of a prepared text, discuss the merits of the block operation, and then set a structured writing task where major changes to the text are required during the development of the writing.

The phenomenon of using Returns at the end of every line rather than adjusting margins may be explained in terms of the pencil and paper analogy. When writing manually, the writer decides when to start a new line and this is not easily assimilated with the word processor's ability to control line length. One approach to this may be to suggest an alternative analogue, for example choosing a narrower sheet of paper. Carroll [9] suggests that alternative analogues may not be helpful and it may be better to create a cognitive conflict situation, where the learner is for example asked to change the font size of their text and explain why it no longer fits their manually created margins. This may help them to see a Return as just another character in the text, albeit an invisible one.

The failure to use a straight line tool when drawing with a graphics program can also be explained by a pencil and paper analogy. Children are expected to use a ruler in certain drawing situations (particularly mathematics), but not in others (such as art), and this distinction is transferred to IT tools. Furthermore, freehand drawing is used with young children to develop mouse skills; this automatized procedure must be overridden. The evaluation of drawings produced in different ways should help to develop the learner's ability to choose appropriate techniques.

There is no good nonelectronic analogy for a spreadsheet model. The observed phenomenon of entry of evaluated results rather than formulas can be explained simply in terms of an inadequate CR of a spreadsheet. The CR merely represents a table of values as seen on the screen. So learners create a grid of numbers to fit the task requirements using familiar calculating methods. The use of variables and formulas requires a well-developed CR of algebra. How linking of these two Crs should be fostered is not yet clear. In our work we want to establish whether skills in spreadsheet modelling help with the development of algebraic concepts. The National Curriculum requirement that initial work in computer modelling should comprise exploration and adaptation of prepared models is likely to help learners develop a CR which incorporates the idea of stored relationships. 


\section{CONCLUSION}

This simplified version of cognitive theories of learning has considerable explanatory power for the development of IT capability, yet it may be quite easily understood and used by teachers. The learning of IT may be improved if teachers first of all recognize the naive conceptions which their students bring in the form of analogies. They should provide structured tasks designed to stimulate conceptual development, and provide procedural instruction (through intervention or reference material) only when necessary. They should also recognize that learners do not always realize when they need instruction, and so teachers should challenge their students' ineffective methods and encourage them to be aware of their learning needs and achievements in addition to focusing on the desired outcome of the task. For those common IT techniques which do not have familiar manual analogues, sufficient practice should be given for them to be automatized. Learners' cognitive resources are then released for higher order thinking and the development of concepts in the other subjects to which IT is applied. Formative assessment should be designed to probe conceptual understanding rather than mere procedural skill; written tests are not appropriate for this purpose as use of computers is required.

Many teachers have themselves mental models which are inadequate for effective use of IT and for helping their students learn and apply IT effectively. Also specialist IT teachers who are needed to help students progress beyond a basic level of capability, need to continually update their own knowledge and skills. Teachers should select appropriate methods for developing their own skills. The mental models approach provides a framework for their learning.

\section{REFERENCES}

1. DES/WO (1990) Technology in the National Curriculum. HMSO, London.

2. Birnbaum, I. (1989) Information Technology and the National Curriculum: some fundamental issues. Resource, Doncaster.

3. Kennewell, S. E. (1993) Changes in strategies for teaching and learning Information Technology across primary/secondary transfer. University College of Swansea Department of Education, Swansea.

4. Cardinale, L. A. (1991) Mental models in action. The Computing Teacher, 1991 (August/September), pp. 16-20. 
5. Anderson, J. R. (1987) Skill acquisition: compilation of weak-method problem solutions. Psychological Review, 94 (2) pp. 192-210.

6. Norman, D. A. (1983) Some observations on mental models, in Mental Models. (eds Gentner, D. and Stevens, A. L.), Lawrence Erlbaum Associates, Hillsdale NJ, pp. 4-14.

7. Bell, A. W. (1993) Principles for the design of teaching. Educational Studies in Mathematics, 24 (1) pp. 5-34.

8. Vygotsky, L. S. (1978) Mind in Society. Harvard University Press, London.

9. Carroll, J. M. and Rosson, M. B. (1987) The paradox of the active user. In: Interfacing Thought (ed Carroll, J. M.), MIT Press, Cambridge Mass., pp. 80-111.

10. Watson, D. M. (1993) The Impact Report. DFE \& Kings College, London. 